\title{
路网信息不完备条件下的动态最短路搜索
}

\author{
龙科军 ${ }^{1,2}$, Lee D. $\mathrm{HAN}^{1,3}$, 王赛政 ${ }^{1,2}$ \\ (1. 长沙理工大学 交通运输工程学院, 长沙 410114；2. 长沙理工大学 道路灾变防治及交通安全教育部工程研究中心, 长沙 410114; \\ 3. 田纳西大学 土木及环境工程系,田纳西 诺克斯维尔 37996-2010,美国) \\ (Lonkj@ sohu.com)
}

摘 要: 针对路网信息不完备性、路网结构特征和驾驶员习惯等因素,研究最短路搜索问题。提出以全局规划和 局部规划相结合的动态最短路混合规划方法: 全局规划中, 基于参数 $d / l$ (起终点距离 $d$ 与平均路段长度 $l$ 之比), 确定 路径搜索区域的椭圆方程,运用 Dijkstra 算法生成静态的全局最短路径; 局部规划中,结合路网结构特征、突发事件影 响范围,提出改进 Bug 算法, 以避免车辆进入全局最短路径上发生的紧急事件或严重堵塞区域,实现动态诱导。仿真 实验结果表明,混合规划方法能在路网信息不完备条件下实现最短路径动态诱导,有效避开拥堵区域。

关键词: 智能交通系统; 最短路搜索;全局规划; 局部规划; 不完备信息

中图分类号: TP301 文献标志码: A

\section{Shortest path search in road network with incomplete information}

\author{
LONG Ke-jun ${ }^{1,2}$, Lee D. HAN ${ }^{1,3}$, WANG Sai-zheng ${ }^{1,2}$ \\ (1. College of Traffic and Transportation Engineering, Changsha University of Science and Technology, Changsha Hunan 410114, China; \\ 2. Highway Disaster Prevention and Traffic Safety Engineering Research Center of Ministry of Education, \\ Changsha University of Science and Technology, Changsha Hunan 410114, China; \\ 3. Department of Civil and Environmental Engineering, University of Tennessee, Knoxville Tennessee 37996-2010, USA)
}

Abstract: Concerning the incomplete information, structural characteristics and driver's habit, issue of the shortest path search in road network was studied. A mixed programming was proposed combining the global and local planning. During global planning, an index $d / l$ was introduced which represented the ratio of Origin-Destination (OD) distance to the average link length, elliptical minimal search area was determined by $d / l$, and the initial global shortest path was calculated by Dijkstra algorithm. During local planning, an improved Bug algorithm was introduced based on network structure and influence range of unexpected events, and the congested or hazard region along the global planned path was avoid. The simulated experimental results indicate that the mixed planning can search the shortest path dynamically in road network with incomplete information and evade from congestion efficiently.

Key words: intelligent transportation system; shortest path search; global planning; local planning; incomplete information

\section{0 引言}

国内外关于最短路径搜索的研究已有许多成果,这些研 究主要针对数学规划 (如图论) 和计算机网络领域,在实际的 道路交通网络应用层面,最短路搜索问题必须考虑路网动态 变化和驾驶员行为的特性与要求,如:路网信息的可获得程 度、驾驶员在路网中寻找最短路径的思维特征等。因此,从路 网特性和驾驶员常规思维特征出发,提出合理、适用的最短路 径搜索方法,对于城市交通诱导、智能交通管理极为重要。

路网最短路径搜索问题特性和要求,重点在 3 方面。

1) 路网中交通流实时动态变化, 各路段的行驶时间为随 机变量,同时,路段上突发事件 (如: 交通事故) 的发生也是不 确定的。

2) 路网实时信息的不完备性, 在整个路网内安装全范围 的交通流检测系统不仅经济上不可行,而且对于大多数智能 交通系统应用上也是不必要的 ${ }^{[1] 225}$ 。现有的关于最短路径算
法大多基于完整的信息,在应用于实际路网最短路径搜索时 无疑具有缺陷。如何在有限的路网信息基础上, 以最小代价 来获得最短路径尤为关键。

3) 驾驶员经验和常规思维对路径搜索提出了要求: 驾驶 员在选择最短路径时,不可能扫描整个路网面积,而是主要搜 索起终点连线两侧的部分路网, 这为最短路径搜索限定了某 个有效区域,从而减少大量不必要的节点和路段搜索。

现有典型的最短路算法包括:标号法、标号修正算法、k最短路径算法、启发式算法、分层路径搜索算法等。Dijkstra 算法(标号法)的基本思想是基于加权图 (权重为非负)的单 源最短路径搜索算法, 以起始节点为中心向外层扩展, 每次取 距离最短的邻接节点作为下一次搜索的节点,直至扩展目标 到目标节点位置 ${ }^{[2] 21}$, Dijkstra 算法的最大缺陷是其遍历计算, 需要较大的计算量和储存空间。

Bellman-Ford 算法是一种标号修正算法,能在更普遍情况 下 (存在负权边) 解决单源点最短路问题, 基本思想为: 给定带

收稿日期:2010-09-25; 修回日期:2010-11-17。

基金项目:国家“十一五”科技支撑计划项目 (2009BAG13A02); 湖南省科学技术厅科技计划重点项目(2010WK4001)。

作者简介: 龙科军 $(1974-)$, 男, 湖南双峰人,副教授,博士, 主要研究方向:智能交通、交通安全与行为; Lee D. HAN $(1963-)$, 男, 美国田 纳西州人,副教授,博士,主要研究方向:智能交通、交通数据融合与挖掘; 王赛政 $(1985-)$, 男,湖南益阳人,硕士研究生,主要研究方向: 智能 交通。 
权图 $G$, 对图运行 Bellman-Ford算法得到一个布尔值结果, 表明 图 $G$ 中是否存在一个从起点可达的负权回路, 如果不存在这样 的回路,将给出从起点到图 $G$ 的任意节点的最短路径 ${ }^{[3]}$ 。

$k$ - 最短路径算法不仅考虑最短路, 也考虑次短路径, 同时 求出长度从小到大排列的 $k$ 条最短路径, 以便用户根据自己 习惯和喜好进行选择, 同时, 当最短路径不可行时为用户提供 更现实、较好的替代方案 ${ }^{[4] 821}$ 。

启发式算法包括局部择优搜索、最好优先搜索等, 其代表 为 $\mathrm{A} *$ 算法 ${ }^{[4] 820}$ 。 $\mathrm{A} *$ 算法是一个目标定向搜索算法, 其优点 是把 Dijkstra 算法(靠近起点的节点)和最佳优先搜索的信息 块结合起来, 代价函数 $f()=g()+h()$, 其中 $g()$ 为起点至 当前节点 $i$ 的代价, $h()$ 为当前节点 $i$ 到目标节点的启发式估 计代价, 每次进行循环计算时, 检查 $f($ ) 最小的节点 $i$, 并形成 最优路径。

分层路径搜索算法 ${ }^{[5]}$ 的基本思想是:假设出行者大多选择 其比较熟悉、道路等级较高、连通性较好的路径, 而不是单纯地 依距离最短, 在算法设计上, 将路网抽象为不同层, 模拟人们实 际经验, 距离目的地越远, 路径偏向于高等级道路, 不同的路网 层之间通过进出口节点相连。分层算法的缺点是地理信息、连 接信息和某些属性重复存储, 导致存储空间过度增长。

总结起来, 现有的算法均有各自优点和适应条件,但在应 用于路网交通诱导时存在不足: 不能充分考虑路网状态实时 变化,难以应对路网中的突发事件; 不能完整体现驾驶员在路 径选择上的偏好, 导致最终的路径方案难以得到驾驶员的认 可。

基于此,本文针对路网交通信息可达性、驾驶员司惯和突 发事件随机性等特性, 提出一种全局规划和局部规划相结合 的混合算法, 以提高最短路算法在智能交通管理中的适用性。

\section{1 问题描述和解决方法}

交通诱导主要目的是根据获得的路网交通流信息, 为驾 驶员提供从起点到目的地的最优/最短行驶路径。面向交通 诱导的最短路搜索主要解决三个问题: 真实的道路网络由于 承载交通的实时动态变化、完整路网信息采集难度大,如何在 不完备信息条件下搜索最短路; 驾驶员对诱导路径方案的认 可程度必然影响到路径方案质量, 也就是说, 最短路径搜索方 法必须考虑到驾驶员的行为妇惯; 路网中突发的事件不可预 测, 路径搜索必须具备应对突发事件的能力。

基于此,本文结合驾驶员习惯,提出一种不完备信息条件 下的最短路径搜索混合算法 ${ }^{[6]}$, 如图 1 所示, 系统由全局路径 规划和局部规划两部分组成。全局规划生成初始的全局最短 路径, 当行驶过程中某路段/节点出现突发事件引发拥堵时, 利用局部规划来绕避拥堵,并重新回归全局最短路径。

全局路径规划 根据给定的路网结构和交通流分布信 息, 结合驾驶员经验和习惯, 从起点和目的地之间生成一条最 短路径; 由于路网交通状态信息难以完整获得,驾驶员在选择 路径时的习惯趋向于起终点连线附近, 针对该两个特征, 全局 路径规划算法采用限制搜索区域算法,在起终点连线两侧划 定可能的搜索范围, 应用常规算法 (如: Dijkstra 算法) 获得最 短路径。

局部规划 路网状态实时动态变化, 全局规划不能包含 路网的所有动态信息(如:突发的交通事故等), 因此,车辆在 依全局规划路径行驶过程中必须依靠局部规划来应对突发事
件; 基于此,采用局部规划算法 (如:Bug 算法)跟踪全局路径、 避开堵塞路段,修正全局路径,最终到达目的地。

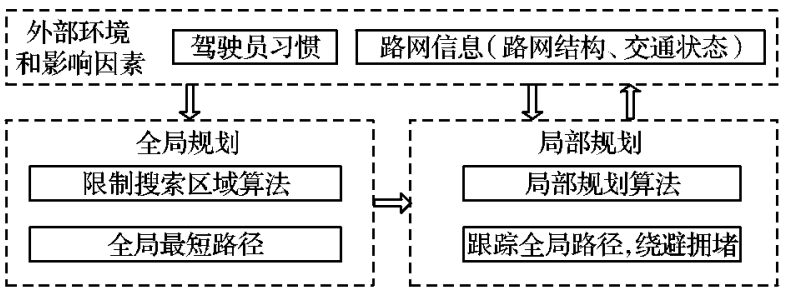

图 1 不完备信息下的混合规划方法

2 限制搜索区域全局最短路搜索

驾驶员从路网中搜索一条最短路径时,不需要对整个路 网的每条路段进行考查,一般尽量选择与目的节点相一致的 方向, 并尽量躲避障碍物。也就是说, 最短路径搜索时, 可以 基于某些规则来删减节点或路段, 以减少搜索范围并提高搜 索速度。过去研究中关于限定捜索区域的方法包括圆形 法 $^{[7]}$ 、椭圆法 ${ }^{[1] 227}$ 、椭圆外切矩阵 ${ }^{[8]}$ 、椭圆内含矩阵 ${ }^{[9]}$ 等, 其 中椭圆法被证明具有 $95 \%$ 的可信度,但是, 椭圆法的难点是： 确定椭圆方程需要对路网中所有出行起终点 (OriginDestination, OD) 对的最短路径进行统计分析来获取,前期准 备工作量巨大，必须采用一种代替方法来解决。

假设出行的起点为 $S$ 到目标节点为 $T$, 根据驾驶员经验 和习惯, 可以限定搜索区域如图 2 中的椭圆, 椭圆方程为:

$$
\frac{\left[x-\left(x_{S}+x_{T}\right) / 2\right]^{2}}{a^{2}}+\frac{\left[y-\left(y_{S}+y_{T}\right) / 2\right]^{2}}{b^{2}}=1
$$

其中: $x 、 y$ 为椭圆任意点的坐标, $x_{S} 、 y_{S} 、 x_{T} 、 y_{T}$ 分别为起终点 $S$ 、 $T$ 的坐标, $a 、 b$ 分别为椭圆的长轴和短轴。

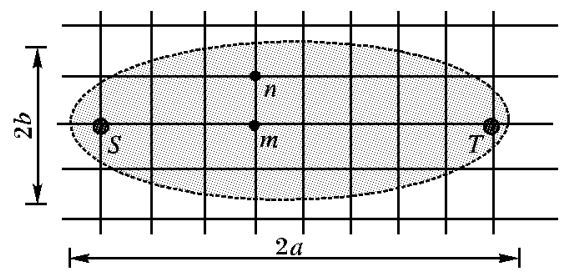

图 2 椭圆限制搜索区域图例

显然, $a 、 b$ 值决定了搜索范围的大小,如何减少搜索工作 量,限定最小的椭圆面积是关键,过去的研究大多推荐采用统 计方法来确定椭圆方程, 这无疑增加了前期工作量, 本文将从 路网结构特征来确定搜索椭圆的形状。

搜索椭圆的大小不仅受驾驶员习惯和行为的影响, 也受 到路网结构尤其是路段长度的影响。简单地说,图 2 中椭圆短 轴 $b$ 的大小必然受与 $S-T$ 连线相交的路段 $(m, n)$ 长度的影 响, $(m, n)$ 长度越大, $b$ 值也将越大。

计算起终点的直线距离 $d$ 为:

$d=\sqrt{\left(x_{S}-x_{T}\right)^{2}+\left(y_{S}-y_{T}\right)^{2}}$

对于结构相对规则的路网 (如: 方格网状), 假设路网的 平均路段长度为 $l, d$ 与 $l$ 的比较关系直接影响椭圆参数 $a$ 和 $b$, 在此,本文采用 $d / l$ 来表示。直观可以得到, $d / l$ 与椭圆形状 之间的关系为: $d / l$ 较小时,表明起终点距离较近, 路段长度 与起终点距离相差不大时,椭圆更趋向于圆形; 随着 $d / l$ 增 大, 表明起终点距离较远, 起终点距离远超过路段长度, 此时, 椭圆更扁长。基于此思想, 可以得到椭圆长轴 $a$ 、短轴 $b$ 与 $d / l$ 的关系, 在此, 结合 Lee D. HAN $(2006)^{[1] 230}$ 的成果, 经变形得 到: 


$$
\left\{\begin{array}{l}
a=0.51 d \\
b=\frac{2 d}{3(d / l)^{0.3546}}
\end{array}\right.
$$

在获得了限制搜索区域的基础上, 运用 Dijkstra 算法进 行路径树生成, 并判断新拓展的节点是否位于椭圆限制区域 内, 即可得到全局最短路径, 鉴于 Dijkstra 算法为非常成熟的 算法,在相关文献 $[2,4,10]$ 均有介绍,在此不再赘述。

\section{3 局部规划路径}

路网交通流实时演化, 由于建设和运营成本限制, 现有的 路网交通流检测系统不能完整地把每条道路和节点的交通状 态实时采集和传输, 事实上, 驾驶员是在一个信息不完备的路 网中工作, 当路网中出现紧急交通事件时, 诱导系统和驾驶员 获取的信息往往不完备或者出现延迟,因此,全局规划不能应 对该问题,需要局部规划来解决。

给定路网 $G(N, A)$, 基于全局规划的最优路径节点集为 $\operatorname{Pr}(M)$, 驾驶员沿着路径 $\operatorname{Pr}(M)$ 行驶, 当路径中某路段 $(i, j)$ 发生紧急事件不能通行时, 必须选择事件影响区的相邻道路 来绕行。同时, 为了保证前进方向与目的地一致,绕行的路径 往往近邻影响区边界, 该特征与 Bug 算法 ${ }^{[11]}$ 思想非常类似, 下面结合驾驶员和路网结构特征, 采用改进 Bug 算法来实现 局部路径规划。

基于驾驶员和路网结构特征的改进 Bug 算法基本流程如 下。

步骤 1 车辆沿着全局规划路径 $\operatorname{Pr}(M)$ 向目标节点 $T$ 行 驶,直到下面情况之一出现:

1) 到达目标节点 $T$, 停止搜索;

2) 车辆到达节点 $i$ 时, 路段 $(i, j)$ 已出现障碍物 (如: 突发 事件、交通拥堵), 转至步骤 2 。

步骤 2 预测或通过获取信息, 得到障碍物清除剩余时 间 $\Delta t$ 是否大于某阈值 $\tau$ :

1) 如果 $\Delta t \geqslant \tau$, 则转至步骤 3 ;

2) 否则, 继续沿主路径 $\operatorname{Pr}(M)$ 行驶。

步骤3 生成障碍物边界节点集 $R$ (如图 3 ) : 将当前节点 $i$ 置人 $R$ 中, 令 $i \rightarrow R(1)$, 从邻接顶点表中读取 $i$ 的邻接节点, 如果邻接节点满足两个条件 (1) 距离障碍物区域 $B$ 中心 $O$ 最 短, 即: $d_{\min }(i+1, O)$; (2) 不在障碍物区域之内), 则为边界节 点,置人 $R$ 中, 依次类推

步骤 4 沿着障碍物边界 $R$ 行驶, 直到下面情况之一出现:

1) 当车辆到达目标节点 $T$, 即 $R(k)=T$, 则结束;

2 ) 当车辆在边界节点 $R(k)$ 回到主路, 即 $R(k) \in$
$\operatorname{Pr}(M)$, 且从节点 $R(k)$ 至目标节点距离比 $i$ 点小, 即 $d(R(k)$, $T)<d(i, T)$, 则转至步骤 1 。

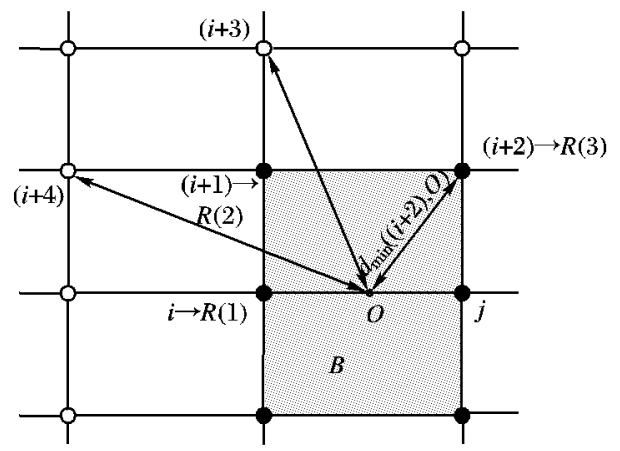

图 3 突发事件影响区域边界节点生成图示

4 案例仿真

以 C\#. net 为开发环境, 利用 MAPX 控件 ${ }^{[12]}$,构建实验仿 真平台, 并以某城市片区的实际路网开展仿真实验, 实验以基 于周期来规划:

1) 根据路网当前信息,生成全局初始最短路径;

2) 在系统内设定规划周期;

3)规划周期内, 更新路网交通信息, 获取突发事件、严重 堵塞信息 (事件类型、发生地点、事件清除事件等), 定位车辆 当前位置;

4)运用局部规划, 更新局部路径, 绕避事件影响区域, 回 归全局路径, 直到目的地。

起始节点编号为 1 , 目标节点为 18 , 车辆行驶过程中, 路 段 $(9,10)$ 和 $(14,18)$ 上发生突发事件,假设拥堵范围没有超 过路段的两端点, 仿真实验的过程如图 4(a) (c)。

图 4(a) 由全局规划生成初始的最短路径, 车辆行驶过程 中定期获取路网动态信息, 当车辆行至障碍物附近时通过局 部规划绕行, 重新生成路径, 绕开拥堵点, 直到目的地。将本 研究算法与静态规划算法的效率进行比较,如表 1 。

\section{表 1 静态和动态规划路径效益对比}

\begin{tabular}{cccc}
\hline 步骤 & 新路径代价/s & 旧路径代价/s & 节省代价比例 $\%$ \\
\hline 第 1 步 & 316 & 316 & 0 \\
第 2 步 & 405 & 405 & 0 \\
第 3 步 & 435 & 435 & 0 \\
第 4 步 & 440 & 478 & 7 \\
第 5 步 & 488 & 566 & 14 \\
第6 步 & 433 & 500 & 13 \\
$\vdots$ & $\vdots$ & $\vdots$ & $\vdots$ \\
\hline
\end{tabular}

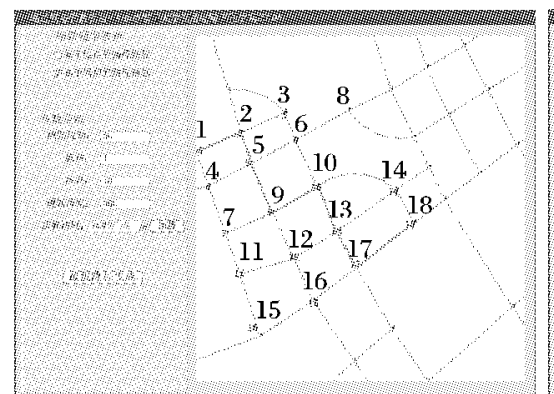

(a) 生成全局初始最短路径

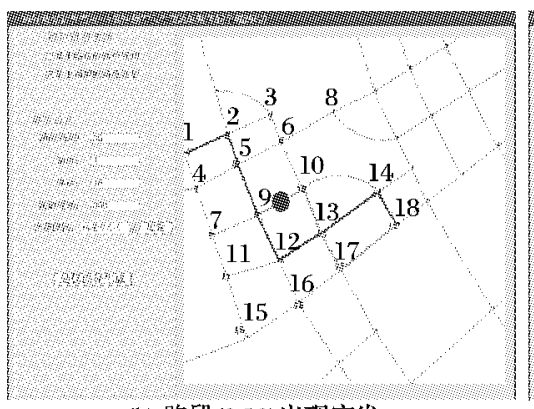

(b) 路段 $(9,10)$ 出现突发 事件时重新规划路径

图 4 路网动态路径规划仿真实验结果

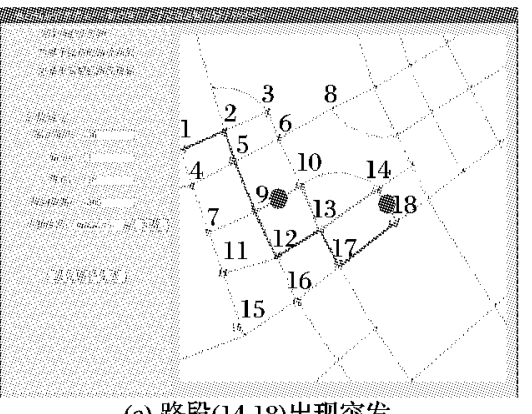

(c) 路段 $(14,18)$ 出现灾发 事件时再次规划路径 


\section{表 3 MPCA 与 MECA 收敛所需的平均函数评价次数的比较}

\begin{tabular}{cccc}
\hline \multirow{2}{*}{ 函数 } & \multirow{2}{*}{ 函数值 } & \multicolumn{2}{c}{ 平均函数评价次数 } \\
\cline { 3 - 4 }$F 06$ & 0 & 6852 & MPCA \\
$F 08$ & -12569.4866 & 20249 & 7960 \\
$F 09$ & 0 & 32171 & 17824 \\
$F 10$ & 0 & 77026 & - \\
$F 12$ & $1.571 \times 10^{-32}$ & 56547 & 9299 \\
$F 13$ & $1.350 \times 10^{-32}$ & 59233 & 9619 \\
$F 15$ & -78.3323314 & 46937 & 12322 \\
\hline
\end{tabular}

\subsection{MPCA 的收敛性}

文献 $[10]$ 已经证明, 凡是保留上代最优个体在种群中的 算法, 都收玫到全局最优解。MPCA 对每个种群每次都保留 最优个体强配子在子代中。所以, 根据文献 $[10]$ 的结论, MPCA 收敛到全局最优解。

\section{4 结语}

多种群协同进化数值优化算法的思想来自于生物学的杂 种优势理论。将这种理论应用到进化计算中的原则就是要尽 可能保持种群的纯洁性与差异性, 使种群朝着多样性的方向 发展, 从而提高进化算法的抗早熟能力, 更有效地搜索全局最 优解。本文提出的 MPCA 在不同类型的种群中采用了不同的 算子,并使用了大小栖息地与跳跃基因来进行全局最优解的 广度与深度搜索。通过对 15 个高维标准测试函数的实验以 及与其他优质算法的对比表明, MPCA 在收玫性、运算效率、 全局最优解精度方面都有较好的表现。

\section{(上接第 653 页)}

仿真结果表明,动态规划算法能依路网实时信息调整路 径, 绕避拥堵区段, 通过局部规划路径调整, 行车效率得到有 效提高。

\section{5 结语}

1) 路网信息不完备、路网结构特征和驾驶员习惯是最短 路搜索面临的关键问题, 从这三个方面切人开展最短路研究 是必要的。

2) 路网信息实时、动态变化, 而由于建设运营成本限制, 系统不可能获得完备的路网信息, 采用全局规划和局部规划 相结合的方法, 可以适应路网不完备信息条件下最短路搜索 问题。

3) 椭圆限制搜索区域的最短路搜索算法不仅符合驾驶 员习惯和行为,而且能适应路网不完备信息的环境; 基于驾驶 员行为的改进 Bug 算法能有效绕避路网中的突发事件和拥堵 点段。

4) 本文有待进一步研究需求:算法效率有待进一步检 验; 局部规划中, 驾驶员对拥堵区的态度有待进一步深人研 究,包括拥堵区范围和清除时间。

\section{参考文献：}

[1] HAN L D, WANG HUI, MACKEY W F. Finding shortest paths under time-bandwidth constraints by using elliptical minimal search area $[\mathrm{J}]$. Transportation Research Record: Journal of the Transportation Research Board, 2006, 1977: 225 -233.

\section{参考文献:}

[1] ZHONG WEICAI, LIU JING, XUE MINGZHI, et al. A multi-Agent genetic algorithm for global numerical optimization [J]. IEEE Transactions on Systems, Man, and Cybernetics - Part B: Cybernetics, 2004, 34(2) : $1128-1141$.

[2] 陈皓, 崔杜武, 崔颖安, 等. 族群进化算法 [J]. 软件学报, 2010, 21 (5) : $978-990$.

[3] 杨业华. 普通遗传学[ M] . 2 版. 北京: 高等教育出版社, 2008: 298 -300 .

[4] 慕彩红, 焦李成, 刘逸. M-精英协同进化数值优化算法 $[\mathrm{J}]$. 软件 学报, 2009, 20(11): 2925-2938.

[5] LIU JING, ZHONG WEICAI, JIAO LICHENG. An organizational evolutionary algorithm for numerical optimization [J], IEEE Transactions on Systems, Man, and Cybernetics - Part B: Cybernetics, 2007, 37(4): $1052-1064$.

[6] van den BERGH F, ENGELBRECHT A P. A cooperative approach to particle swarm optimization [ J]. IEEE Transactions on Evolutionary Computation, 2004, 8(3): 225-239.

[7] YAO XIN, LIU YONG, LIN GUANGMING. Evolutionary programming made faster [J]. IEEE Transactions on Evolutionary Computation, 1999, 3(2): $82-102$.

[8] LEUNG Y W, WANG Y P. An orthogonal genetic algorithm with quantization for global numerical optimization [J]. IEEE Transactions on Evolutionary Computation, 2001, 5(1): $41-53$.

[9] SHANG YUNWEN, QIU YUHUANG. A note on the extended rosenbrock function [ J]. IEEE Transactions on Evolutionary Computation, $2006,14(1): 119-126$.

[10] RUDOLPH G. Convergence analysis of canonical genetic algorithms [J]. IEEE Transactions on Neural Networks, 1994, 5(1): $96-101$.
[2] NORDBECK S, RYSTEDT B. Computer cttography sortest rute pograms [M]. Sweden: The Royal University of Lund, 1969: 18 -30.

[3] 陈华容, 张崇富. Bellman-Ford 算法的改进研究 [J]. 电子科技大 学学报, 2006,35(2): 211 -213.

[4] 李威武, 王慧, 钱积新. 智能交通系统中路径诱导算法研究进展 [J]. 浙江大学学报: 工学版, 2005, 39(6):819-825.

[5] 苗洋, 陈奇. 嵌人式环境中分层路径规划算法的改进 [J]. 计算机 工程, 2010,36(14) : 243-245.

[6] 郑宏, 王景川, 陈卫东. 基于地图的移动机器人自定位与导航系 统 [J]. 机器人, 2007, 29(4) : 397 -402.

[7] HAN L D, MACKEY W F. Research issues in intelligent transportation systems $[\mathrm{C}] / /$ The International Workshop on Geographic Information Systems for Transportation (GIS-T) and Intelligent Transportation Systems ( ITS) . Hong Kong: The Chinese University of Hong Kong, 1999: $101-108$.

[8] 陆锋, 卢冬梅, 崔伟宏. 道路网络限制搜索区域时间最短路径算 法 [J]. 中国图象图形学报, 1999,4(10):849-853.

[9] 王亚文, 汪西莉, 曹菡, 等.一种动态限制搜索区域的最短路径规 划算法 [J]. 计算机应用研究, 2007, 24(7) :89-91.

[10] 任子晖, 王坚. 紧急事件的动态交通流模型及双向动态最短路诱 导算法 [J]. 计算机应用, 2008, 28(11) : 2955 -2957.

[11] KAMON I, RIVLIN E. Sensory-based motion planning with global proofs [J] . IEEE Transactions on Robotics and Automation, 1997, $13(6): 814-822$.

[12] 韩得. 地理信息系统开发: MapObjects 方法 [ M]. 武汉: 武汉大学 出版社, 2004: $65-78$. 